\title{
LUT
}

University

\section{Adaptive MIMO Pole Placement Control for Commissioning of a Rotor System with Active Magnetic Bearings}

Nevaranta Niko, Jaatinen Pekko, Vuojolainen Jouni, Sillanpää Teemu, Pyrhönen Olli

This is a Author's accepted manuscript (AAM) version of a publication

published by Elsevier

in Mechatronics

DOI: 10.1016/j.mechatronics.2019.102313

Copyright of the original publication: (c) 2019 Elsevier Ltd.

Please cite the publication as follows:

Nevaranta, N., Jaatinen, P., Vuojolainen, J., Sillanpää, T., Pyrhönen, O. (2019). Adaptive MIMO Pole Placement Control for Commissioning of a Rotor System with Active Magnetic Bearings. Mechatronics, vol. 65. DOI: 10.1016/j.mechatronics.2019.102313

This is a parallel published version of an original publication. This version can differ from the original published article. 


\title{
Adaptive MIMO Pole Placement Control for Commissioning of a Rotor System with Active Magnetic Bearings ${ }^{\star}$
}

\author{
Niko Nevaranta*,1, Pekko Jaatinen, Jouni Vuojolainen, Teemu Sillanpää and Olli Pyrhönen \\ LUT University, Finland
}

\section{ARTICLE INFO}

Keywords:

Active Magnetic Bearing (AMB)

Adaptive control

Commissioning

Magnetic levitation

Rotor system

\begin{abstract}
A B S TRACT
Active magnetic bearing (AMB) supported rotor systems require advanced control strategies to meet the increased performance requirements of more and more demanding applications. To meet the particular requirements for the performance under changing dynamics, an adaptive control structure is a task worth pursuing. This paper studies adaptive multi-input multi-output (MIMO) pole placement applied to the control commissioning of an AMB-supported rotor system. The control tuning approach is based on a rigid body model, and the parameter estimation is carried out with a recursive extended least squares (RELS) based algorithm for the MIMO system. The proposed approach is studied by simulations and validated with both a 2-degree-of-freedom (2-DOF) and a 4-DOF AMB system.
\end{abstract}

\section{Introduction}

Active magnetic bearings (AMBs) are replacing conventional bearings in modern industrial high-speed motor systems, and they are used in applications that have become increasingly demanding over the past few years and that require advanced control strategies to handle the highly dynamic system under disturbances. Despite the extensive development of the control laws for AMBs, the self-tuning and adaptive laws have not been widely addressed in the literature. Naturally, one of the main reasons for this is related to the complex and unstable system dynamics with multipleinputs multiple-outputs (MIMO).

Typically, the initial control synthesis of a high-speed motor with a magnetically supported rotor system is based on the modeled dynamics of the AMBs combined with the flexible rotor system obtained from the analytical tools applied in the design phase[1]. After a stabilizing controller for levitation has been obtained, the control design procedure turns into an iterative identification of a control problem. As the time-consuming commissioning phase often requires a skilled expert, there is a demand for methods and tools that could (semi)automatize the commissioning process. Hence, a self-tuning controller structure with proper adaptive functions is worth pursuing in the case of AMB-supported rotor systems, which can be found in several applications such as gas turbines, compressors, and turbomachinery, to name but a few. The second important aspect of using an adaptive control law is related to the possible process changes, but this problem is naturally application dependent.

There are several studies focusing on the adaptive compensation functions [2], [3], [4], [5] yet only a few studies address the self-tuning or adaptive control of an AMB system. In [6], an adaptive control law has been proposed for a twodegree-of-freedom (2-DOF) AMB system that is capable of

${ }^{\star}$ This document include results of the research project Intelligent Energy Efficient High-Speed Drive funded by the Business Finland.

*Corresponding author

niko.nevaranta@lut.fi (N. Nevaranta)

ORCID(s): $0000-0002-9766-0675$ (N. Nevaranta) stabilizing the closed-loop system under an unknown load or unbalance changes. Similarly, in [7], an adaptive control law has been proposed for a 1-DOF system that is based on a backstepping controller with an observer. Another approach has been introduced in [8], where an adaptive state space control is derived for a 4-DOF AMB system using an innovations model in the state estimation routine. The proposed approach, however, does not properly handle the estimation of the parameters if the routine is not initialized correctly, and thus, suffers from some stability issues. A similar idea has been introduced in [9], where a least squares (LS) based estimator is used to estimate the cross-coupling stiffness of a rigid rotor. In [10], a baseline PID controller combined with an adaptive control law to compensate the changing crosscoupling stiffness is proposed. The reported studies are numerical ones obtained by simulations, and so far, there are only a few studies that have shown the implementation of the approaches in an actual AMB application. In [11], an all-coefficient adaptive control law for a flexible rotor system has been introduced and experimentally validated on a test rig.

Motivated by the features of the adaptive state space controller approach reported in [8], which is based on [12], the objective of this paper is to propose an adaptive state space control law for an AMB system. The approach presented here is different from [8] in four respects. First, a separate state estimation routine is considered that updates the estimator with parameters provided by the identification algorithm. This is a more stable approach, as is stated in [13]. Second, instead of an innovations-model-based parameter estimation routine, a recursive extended LS (RELS) algorithm [14] is applied to the MIMO system identification to reduce the bias in the estimates. More importantly, instead of placing the poles in the same location, as is typically proposed in the literature [8], [15], a design-polynomial-based approach is considered to achieve more specific control objectives. Finally, the approach presented here is based on the adaptation of the whole control structure, whereas previous papers have mostly focused on the adaptive cross-coupling 
terms. The proposed adaptive state space control approach is derived for both 2-DOF and 4-DOF AMB systems and experimentally validated on laboratory test rigs.

\section{Problem Statement}

First, an adaptive pole-placement-based control law is derived for a 2-DOF AMB system, and after that, it is extended to a 4-DOF system. The derivation is started from the modeling of the 2-DOF system, and a controllable canonical state space model is presented. After that, a recursive parameter estimation routine for the MIMO system is derived, and finally, the state space control structure is given.

\subsection{Modeling of a 2-DOF AMB System}

By considering the attractive force generated by a pair of two opposite horseshoe electromagnets depicted in Fig. 1 , the force-current dependence of the electromagnets on one coordinate axis (e.g. radial x-axis) can be approximated as follows [16]

$$
F_{b x}=\frac{\mu_{0} N^{2} A_{a i r} \cos (\alpha)}{4}\left(\frac{i_{1, x}^{2}}{\left(l_{0}-x\right)^{2}}-\frac{i_{2, x}^{2}}{\left(l_{0}+x\right)^{2}}\right),
$$

where $\mu_{0}$ is the permeability of a vacuum, $N$ is the number of coil turns, $A_{\text {air }}$ is the cross-sectional area of the pole, $\alpha$ is the force acting angle, $l_{0}$ is the nominal air gap, and $i_{1}$ and $i_{2}$ are the coil currents. Because of the nonlinear dynamics, it is desirable to limit the coil currents and include a linearization with the bias current $i_{\text {bias }}$. The coil currents $i_{1, x}$ and $i_{2, x}$ are then written as

$$
\begin{aligned}
& i_{1, x}=\left\{\begin{array}{ll}
i_{\text {bias }}+i_{c}, & \text { if } i_{c} \geq-i_{\text {bias }} \\
0, & \text { if } i_{c}<-i_{\text {bias }}
\end{array},\right. \\
& i_{2, x}= \begin{cases}i_{\text {bias }}-i_{c}, & \text { if } i_{c} \leq i_{\text {bias }} \\
0, & \text { if } i_{c}>i_{\text {bias }}\end{cases}
\end{aligned}
$$

The force relation in the selected linearization point can be expressed as

$$
F_{b x}=k_{i} i_{x, c}+k_{s} x,
$$

where $k_{i}$ is the current stiffness and $k_{s}$ is the position stiffness, respectively. The equations for the current and position stiffnesses are

$$
\begin{aligned}
& k_{i}=\left.\frac{\partial f}{\partial i_{c}}\right|_{x=0, i_{c}=0}=\frac{\mu_{0} N^{2} i_{\text {bias }} A_{\text {air }} \cos (\alpha)}{l_{0}^{2}}, \\
& k_{s}=\left.\frac{\partial f}{\partial x}\right|_{x=0, i_{c}=0}=\frac{\mu_{0} N^{2} i_{\text {bias }}^{2} A_{\text {air }} \cos (\alpha)}{l_{0}^{3}},
\end{aligned}
$$

where the linearization point is assumed to be the origin $x=0$, and the control current is zero $i_{c}=0$ with the bias

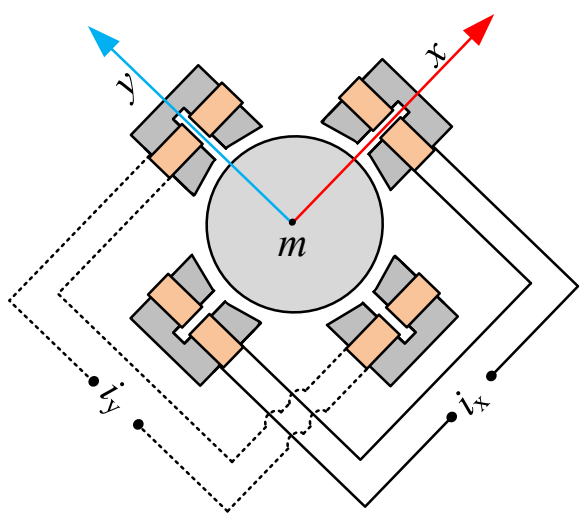

Figure 1: Basic principle of a single mass supported by an active magnetic bearing. The dynamics can be modeled as a 2-DOF system.

current being $i_{\text {bias }}$. The linearized model (4) is linear in a large region, especially when considering $k_{i}$ (5) but also for $k_{x}(6)$ if deviations from the origin are small.

Based on this linearization, the dynamics of a single mass suspended by an AMB depicted in Fig. 1 can be presented with the following state space model

$$
\begin{aligned}
\dot{\mathbf{x}}(t) & =\mathbf{A x}(t)+\mathbf{B u}(t), \\
\mathbf{y}(t) & =\mathbf{C} \mathbf{x}(t),
\end{aligned}
$$

where the state vector $\mathbf{x}=[x, y, \dot{x}, \dot{y}]^{\mathrm{T}}$ is formed from the positions and their derivatives while the input current vector is denoted by $\mathbf{u}=\left[i_{x}, i_{y}\right]^{\mathrm{T}}$. Without any cross-coupling dynamics, the system matrix can be expressed as

$$
\mathbf{A}=\left[\begin{array}{cccc}
0 & 0 & 1 & 0 \\
0 & 0 & 0 & 1 \\
\frac{k_{s}}{m} & 0 & 0 & 0 \\
0 & \frac{k_{s}}{m} & 0 & 0
\end{array}\right],
$$

with the input matrix $B$ and the output matrix $C$

$$
\begin{aligned}
\mathbf{B} & =\left[\begin{array}{ll}
0 & 0 \\
0 & 0 \\
\frac{k_{i}}{m} & 0 \\
0 & \frac{k_{i}}{m}
\end{array}\right], \\
\mathbf{C} & =\left[\begin{array}{llll}
1 & 0 & 0 & 0 \\
0 & 1 & 0 & 0
\end{array}\right],
\end{aligned}
$$

where $m$ is the mass of the rotor, $k_{s}$ is the position stiffness, and $k_{i}$ is the current stiffness. It is noted that the influence of nonconservative forces such as the inner damping in the rotor or the cross-stiffness and cross-damping in fluid seals can be included as cross-coupling terms [17]. By discretizing (8), (9) with the sample time $T_{s}$ by the following matrix transformation, we obtain 


$$
\begin{aligned}
& \boldsymbol{\Phi}=e^{\mathbf{A} \cdot T_{s},} \\
& \boldsymbol{\Gamma}=\left(e^{\mathbf{A} \cdot T_{s}}-\mathbf{I}\right) \cdot \mathbf{A}^{-1} \cdot \mathbf{B},
\end{aligned}
$$

where $\boldsymbol{\Phi}$ and $\boldsymbol{\Gamma}$ are the discretized system matrix and the input matrix, respectively. For pole placement controller design purposes, the state space model is considered in the controller canonical form. It has been found that this form is useful in the design of state feedback laws for MIMO systems by pole placement [8], [12] and thus, it is also considered here. By using the transformation matrix $\mathbf{T}_{c}$, the discretized system matrices (11) can be transformed into canonical ones [18]

$$
\begin{aligned}
& \boldsymbol{\Phi}_{c}=\mathbf{T}_{c} \cdot \boldsymbol{\Phi} \cdot \mathbf{T}_{c}^{-1}, \\
& \boldsymbol{\Gamma}_{c}=\mathbf{T}_{c} \cdot \boldsymbol{\Gamma}, \\
& \mathbf{C}_{c}=\mathbf{C} \cdot \mathbf{T}_{c}^{-1} .
\end{aligned}
$$

This allows to present the system in the following general form

$$
\begin{aligned}
& \boldsymbol{\Phi}_{c}=\left[\begin{array}{cccc}
0 & 1 & 0 & 0 \\
-a_{2}^{\{1,1\}} & -a_{1}^{\{1,1\}} & -a_{2}^{\{1,2\}} & -a_{1}^{\{1,2\}} \\
0 & 0 & 0 & 1 \\
-a_{2}^{\{2,1\}} & -a_{1}^{\{2,1\}} & -a_{2}^{\{2,2\}} & -a_{1}^{\{2,2\}}
\end{array}\right], \\
& \boldsymbol{\Gamma}_{c}=\left[\begin{array}{ll}
0 & 0 \\
1 & 0 \\
0 & 0 \\
0 & 1
\end{array}\right], \\
& \mathbf{C}_{c}=\left[\begin{array}{llll}
c_{2}^{\{1,1\}} & c_{1}^{\{1,1\}} & c_{2}^{\{1,2\}} & c_{1}^{\{1,2\}} \\
c_{2}^{\{2,1\}} & c_{1}^{\{2,1\}} & c_{2}^{\{2,2\}} & c_{1}^{\{2,2\}}
\end{array}\right],
\end{aligned}
$$

where the parameters $a_{1}, \ldots, a_{2}$ and $c_{1}, \ldots, c_{2}$ represent the discrete parameters of the canonical matrices. It can be seen from the state space matrices that the system dynamics is now represented by submatrices resulting in a straightforward general form for pole placement; there are two coupled second-order systems indicated by superscripts 1 and 2 . As the adaptive control algorithm is to be used in an actual high-speed machine, the canonical form must be extended to a 4-DOF AMB system. The canonical form for a larger system can be generalized as $\boldsymbol{\Phi}_{c}=\left\{\boldsymbol{\varphi}^{\{i, j\}}\right\}, \boldsymbol{\Gamma}_{c}=\left\{\boldsymbol{\gamma}^{\{i, j\}}\right\}$ and $\mathbf{C}_{c}=\left\{\mathrm{c}^{T\{i, j\}}\right\}$ with the indices of $i, j=1,2,3,4$ and $i \neq j$ resulting in submatrices in the form

$$
\begin{gathered}
\boldsymbol{\varphi}^{\{i, i\}}=\left[\begin{array}{cc}
0 & 1 \\
-a_{2}^{\{i, i\}} & -a_{1}^{\{i, i\}}
\end{array}\right], \\
\boldsymbol{\varphi}^{\{i, j\}}=\left[\begin{array}{cc}
0 & 0 \\
-a_{2}^{\{i, j\}} & -a_{1}^{\{i, j\}}
\end{array}\right],
\end{gathered}
$$

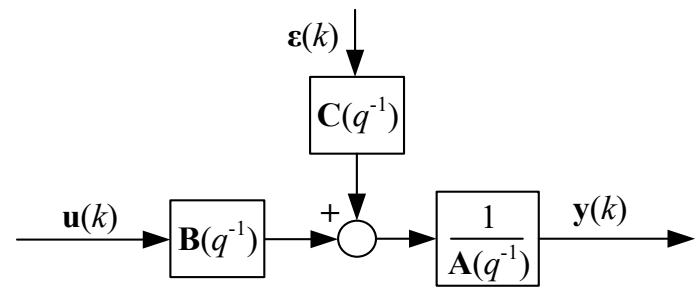

Figure 2: General model structure for the MIMO system modeling considered in the parameter estimation routine. $q^{-1}$ is the backward shift operator.

$$
\begin{aligned}
& \boldsymbol{\gamma}^{\{i, j\}}=\left[\begin{array}{ll}
0 & 0
\end{array}\right]^{\mathrm{T}}, \\
& \boldsymbol{\gamma}^{\{i, i\}}=\left[\begin{array}{ll}
0 & 1
\end{array}\right]^{\mathrm{T}}, \\
& \boldsymbol{c}^{\mathrm{T}\{i, j\}}=\left[\begin{array}{ll}
c_{2}^{\{i, j\}} & c_{1}^{\{i, j\}}
\end{array}\right]^{\mathrm{T}},
\end{aligned}
$$

These matrices are used to build the canonical form of the 4-DOF AMB system considered in this paper. Naturally, the resulting system matrices describe the dynamics of four coupled second-order systems. The 4-DOF model is derived and analyzed by simulations in Section 3 .

\subsection{Parameter estimation algorithm}

Let us assume that the MIMO plant can be modeled with the general model structure depicted in Fig. 2, which has the following polynomial form

$$
\mathbf{A}\left(q^{-1}\right) \mathbf{y}(k)=\mathbf{B}\left(q^{-1}\right) \mathbf{u}(k)+\mathbf{C}\left(q^{-1}\right) \boldsymbol{\epsilon}(k)
$$

where $\mathbf{y}(k)$ is the output vector, $\mathbf{u}(k)$ is the input signal vector, and $\boldsymbol{\epsilon}(k)$ is the unmeasurable noise signal vector. Note that now the notations $\mathbf{A}\left(q^{-1}\right), \mathbf{B}\left(q^{-1}\right)$, and $\mathbf{A}\left(q^{-1}\right)$ represent the polynomials of the general model structure. They can be expressed as

$$
\begin{aligned}
& \mathbf{A}\left(q^{-1}\right)=\mathbf{I}_{p \times p}+\mathbf{A}_{1} q^{-1}+\ldots+\mathbf{A}_{v} q^{-v} \\
& \mathbf{B}\left(q^{-1}\right)=\mathbf{B}_{1} q^{-1}+\ldots+\mathbf{B}_{v} q^{-v} \\
& \mathbf{C}\left(q^{-1}\right)=\mathbf{I}_{p \times p}+\mathbf{C}_{1} q^{-1}+\ldots+\mathbf{C}_{v} q^{-v}
\end{aligned}
$$

where $p$ denotes the number of inputs and $v$ is used to denote the model degree. By considering an autoregressive moving average with an exogenous terms (ARMAX) model for the parameter estimation, the optimal one step ahead predictor (a priori) is formed

$$
\hat{\mathbf{y}}(k+1)=-\hat{\mathbf{A}}(k) \mathbf{y}(k)+\hat{\mathbf{B}}(k) \mathbf{u}(k)+\hat{\mathbf{C}}(k) \mathbf{e}(k+1),
$$

where the polynomials $\hat{\mathbf{A}}, \hat{\mathbf{B}}$, and $\hat{\mathbf{C}}$ denote the estimated ones, and the a priori prediction error is

$$
\mathbf{e}(k+1)=\mathbf{y}(k+1)-\hat{\mathbf{y}}(k+1) .
$$


Naturally, the parametric model is linear to the unknown parameters, but it can be used to fit any linear or nonlinear control system [19]. The predictor can be written in a linear regression form

$$
\hat{\mathbf{y}}(k+1)=\hat{\boldsymbol{\Theta}}^{\mathrm{T}}(k) \boldsymbol{\phi}(k) .
$$

where the parameter matrix $\hat{\boldsymbol{\Theta}}$ and the regression vector $\hat{\boldsymbol{\phi}}$ have the following form

$$
\begin{aligned}
\hat{\boldsymbol{\Theta}} & =\left[\hat{\mathbf{A}}_{1}, \ldots, \hat{\mathbf{A}}_{v}, \hat{\mathbf{B}}_{1}, \ldots, \hat{\mathbf{B}}_{v}, \mathbf{I}_{p}, \hat{\mathbf{C}}_{1}, \ldots, \hat{\mathbf{C}}_{v}\right]^{\mathrm{T}} . \\
\boldsymbol{\phi} & =\left[-\mathbf{y}_{k-1}, . .,-\mathbf{y}_{k-v}, \mathbf{u}_{k-1}, \ldots, \mathbf{u}_{k-v}, \mathbf{e}_{k}, \mathbf{e}_{k-1}, \ldots, \mathbf{e}_{k-v}\right]^{\mathrm{T}} .
\end{aligned}
$$

By considering the time instant $k+1$ when the output $\mathbf{y}(k+$ $1)$ is measured and the parameter estimate is updated, the a posteriori estimate of the output is

$$
\hat{\mathbf{y}}^{\circ}(k+1)=\hat{\boldsymbol{\Theta}}^{\mathrm{T}}(k+1) \boldsymbol{\phi}(k),
$$

and thus, the corresponding a posteriori error is

$$
\hat{\mathbf{e}}^{\circ}(k+1)=\mathbf{y}(k+1)-\hat{\mathbf{y}}^{\circ}(k+1) .
$$

The following recursive least squares algorithm can be used to solve the parameter estimation problem

$$
\hat{\boldsymbol{\Theta}}(k+1)=\hat{\boldsymbol{\Theta}}(k)+\mathbf{F}(k) \boldsymbol{\phi}(k) \mathbf{e}^{\mathrm{T}}(k+1),
$$

$$
\begin{gathered}
\mathbf{e}(k+1)=\frac{\mathbf{e}^{\circ}(k+1)}{1+\boldsymbol{\phi}^{\mathrm{T}}(k) \mathbf{F}(k) \boldsymbol{\phi}(k)}, \\
\mathbf{F}(k+1)=\frac{1}{\lambda_{1}(k)}\left(\mathbf{F}(k)-\frac{\mathbf{F}(k) \boldsymbol{\phi}(k) \boldsymbol{\phi}^{\mathrm{T}}(k) \mathbf{F}(k)}{\frac{\lambda_{1}(k)}{\lambda_{2}(k)}+\boldsymbol{\phi}^{\mathrm{T}}(k) \mathbf{F}(k) \boldsymbol{\phi}(k)}\right),
\end{gathered}
$$

where $\mathbf{F}(k)>0$ is the covariance matrix, and $\lambda_{1}(k)$ and $\lambda_{2}(k)$ are the weighting sequences forming the forgetting factor that has the limits $0<\lambda_{1}(k)<1$ and $0 \leq \lambda_{2}(k)<2$. In this paper, the basic recursive algorithm with a constant forgetting factor is used to validate the pole placement routine, but in the adaptive control law the recursion is regulated by considering a constant trace algorithm, that is, by including a term

$$
\overline{\mathbf{F}}(k+1)=\alpha_{1} \cdot \frac{\mathbf{F}(k)}{\operatorname{tr}(\mathbf{F}(k))}+\alpha_{2} \mathbf{I},
$$

where $\alpha_{1}>0$ and $\alpha_{2} \geq 0$ are the tuning parameters for the constant trace algorithm. The properties of the recursion can be changed by selecting different properties for the forgetting factor and the constant trace [19].

\subsection{State estimation}

To apply the state space control, a full state information is required. Naturally, the parameters and states can be estimated simultaneously, for instance by using the Boostrap algorithms in [20] or by any other approach that estimates states and parameters simultaneously. The basic form of a state estimator can be expressed in the following form

$$
\begin{aligned}
& \hat{\mathbf{x}}(k+1)=\hat{\boldsymbol{\Phi}}_{c} \hat{\mathbf{x}}(k)+\boldsymbol{\Gamma}_{c} \mathbf{u}(k)+\hat{\mathbf{L}}(\mathbf{y}(k)-\hat{\mathbf{y}}(k)), \\
& \hat{\mathbf{y}}(k)=\hat{\mathbf{C}}_{c} \hat{\mathbf{x}}(k),
\end{aligned}
$$

where $\hat{\mathbf{L}}$ is the feedback gain of the estimator and $\hat{\mathbf{x}}$ is the vector of the state estimates. The model (34) can be referred to as an innovations model [21] if it is in a parameterized canonical form, providing an opportunity to estimate $\hat{\boldsymbol{\Phi}}_{c}, \hat{\mathbf{L}}$ and $\hat{\mathbf{C}}_{c}$ simultaneously by using the estimation approach discussed in [8]. Naturally, as a canonical form is applied, the input matrix $\boldsymbol{\Gamma}_{c}$ does not have to be updated. Here, the same canonical form is considered, but the state estimation approach is based on the use of estimated state matrices and the gain $\hat{\mathbf{L}}$ is updated independent of the parameter estimation routine. This has been found to be an effective approach, as stated in [13]. The AMB system is open-loop unstable, and thus, the stabilizing state space control requires a state estimator. Naturally, the estimator dynamics must be designed to be faster than the control dynamics. Hence, the state estimator gain $\hat{\mathbf{L}}$ must be designed in advance, and during the real-time update, the same condition must hold.

\subsection{Pole placement}

In this paper, the pole placement strategy discussed in [12] is considered, but here more sophisticated pole locations are applied. The basic form of the state feedback law is as follows

$$
\mathbf{u}(k)=-\mathbf{K} \cdot \mathbf{x}(k),
$$

where the $\mathbf{K}$ is the state feedback gain. Based on the feedback law, the closed-loop poles are determined by the closedloop system matrix

$$
\boldsymbol{\Phi}_{\mathrm{cl}}=\boldsymbol{\Phi}_{c}-\boldsymbol{\Gamma}_{c} \mathbf{K},
$$

and thus, the resulting characteristic polynomial of the closed loop is $\left|z \mathbf{I}-\boldsymbol{\Phi}_{c}+\boldsymbol{\Gamma}_{c} \mathbf{K}\right|$. The canonical form (13)-(15) provides an opportunity to design the state feedback coefficients directly from the state space model. For example, the following design polynomial can be used to design the controller for each subsystem based on the estimated model in the canonical form

$$
G_{\text {des }}^{\{i, i\}}(z)=z^{2}+\beta_{1}^{\{i, i\}} z+\beta_{2}^{\{i, i\}},
$$

where $\beta_{1}$ and $\beta_{2}$ are used to design the desired performance of the closed-loop controller, that is, the locations of the closed-loop poles. By considering the generalized canonical form (16), (17) the closed-loop system representation (36) can be expressed with the submatrices

$$
\boldsymbol{\varphi}^{\{i, i\}}=\left[\begin{array}{cc}
0 & 1 \\
a_{2}^{\{i, i\}}-k_{2}^{\{i, i\}} & a_{1}^{\{i, i\}}-k_{1}^{\{i, i\}}
\end{array}\right],
$$




$$
\boldsymbol{\varphi}^{\{i, j\}}=\left[\begin{array}{cc}
0 & 0 \\
a_{2}^{\{i, j\}}-k_{2}^{\{i, j\}} & a_{1}^{\{i, j\}}-k_{1}^{\{i, j\}}
\end{array}\right],
$$

when the state feedback gain $\mathbf{K}$ parameterized for the 2-DOF system is

$$
\mathbf{K}=\left[\begin{array}{llll}
k_{2}^{\{1,1\}} & k_{1}^{\{1,1\}} & k_{2}^{\{1,2\}} & k_{1}^{\{1,2\}} \\
k_{2}^{\{2,1\}} & k_{1}^{\{2,1\}} & k_{2}^{\{2,2\}} & k_{1}^{\{2,2\}}
\end{array}\right] .
$$

A straightforward selection for the pole locations is

$$
\begin{aligned}
& k_{m}^{\{i, j\}}=a_{m}^{\{i, j\}}, \\
& k_{m}^{\{i, i\}}=a_{m}^{\{i, i\}}+\beta_{2}^{\{i, i\}}
\end{aligned}
$$

when $m$ is 1,2 in the case of the 2-DOF system. Thus, the desired locations can be designed with (37), and the controller parameters can be redesigned from the identified parameters, that is, adapted to the changes. Obviously, the state feedback has certain limitations, and thus, in order to remove the steady-state error, the state space control structure requires an integral action. This can be obtained by adding integrating states $\mathbf{x}_{\mathbf{I}}$ to the control law

$$
\mathbf{u}(k)=-\left[\begin{array}{ll}
\mathbf{K}, & \mathbf{K}_{\mathrm{I}}
\end{array}\right]\left[\begin{array}{c}
\mathbf{x}(k) \\
\mathbf{x}_{\mathbf{I}}(k)
\end{array}\right],
$$

where $\mathbf{K}_{\mathrm{I}}$ is the integrator gain. In this case, the pole placement is different from (41), as one degree of freedom is added to the control structure. Thus, the design function for the pole locations is a third-order transfer function

$$
G_{\text {des }}^{\{i, i\}}(z)=z^{3}+\beta_{1}^{\{i, i\}} z^{2}+\beta_{2}^{\{i, i\}} z+\beta_{3}^{\{i, i\}} .
$$

Now, the adaptive control structure can be expressed as a general structure as depicted in Fig. 3. The tuning of a pole placement control for an AMB system is often a process of trial-and-error, as in general, it is hard to select locations of the poles for an MIMO system dynamics. For instance, the adaptive approach in [8] was used to place all of the closedloop poles in the same location, more specifically, so that the (43) discrete poles are selected as

$$
z_{1,2,3}=e^{-\sqrt{\frac{k_{s}}{m}} \cdot T_{s}} .
$$

based on the system parameters $m$ and $k_{s}$. This corresponds to an eigenvalue of a spring-damper-mass system with negative stiffness. A similar tuning rule has been applied in [15], [1] for bearingless machines. In this paper, the third-order design polynomial is considered to achieve the desired dynamics for the closed-loop controller

$$
G_{\mathrm{des}}(s)=\frac{1}{s \tau+1} \cdot \frac{\omega_{n}^{2}}{s^{2}+2 \zeta \omega_{n}+\omega_{n}^{2}},
$$

with the following design parameters: time constant $\tau$, natural frequency $\omega_{n}$, and damping $\zeta$. These values are used to design a controller that achieves the desired performance, that is, the closed-loop bandwidth and the maximum sensitivity peak. It is noteworthy that (45) serves as an illustrative initial design function, and thus, the locations of the poles are always trial-and-error tuning procedures.

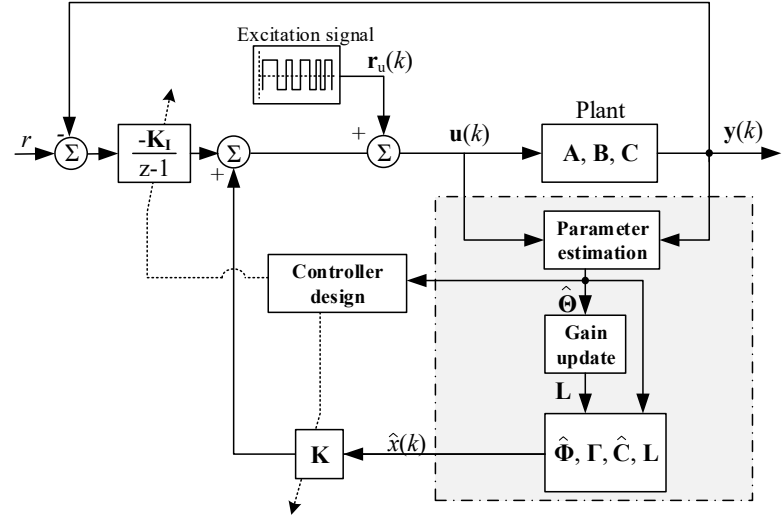

Figure 3: Generalized principle of the adaptive control law. Excitation signals $\mathbf{r}_{u}(k)$ are superposed to the output of the controller to improve the parameter convergence. N.B. The inner current loop is not depicted.

\subsection{Excitation Signal}

In order to obtain valid parameter estimation convergence, the identification approach is supported by artificially generated excitation signals. The persistent excitation signal, viz. a pseudo random binary signal (PRBS), is superposed to the position controller output as shown in Fig. 3. In [22], it has been shown that different excitation signals are valid for AMB system identification. Here, the system has several inputs, and thus, all the inputs should be excited at the same time to guarantee the parameter convergence. In the case of a system with several inputs, the same PRBS can be used if it is time shifted [23] to make them statistically uncorrelated by

$$
r_{\mathrm{u}, n}(t)=A_{n} \cdot u_{\mathrm{PRBS}}\left(t-\theta_{n}\right), \quad n=1,2, \ldots, p,
$$

where $A$ is the amplitude of the signal, and the time shift can be selected as $\theta_{n}=N \cdot T_{\mathrm{sw}} \cdot(n-1) / p$, where $N$ is the length of the signal with the switching time $T_{\mathrm{sw}}$. The PRBS is generated by an 15-cell shift register with a switching time of $T_{\mathrm{sw}}=6 \cdot T_{s}$ when $T_{s}=50 \mu \mathrm{s}$. The amplitude selection will be discussed in the following sections.

\section{Simulations of the adaptive control}

The proposed adaptive control law is studied by simulations by considering a bearingless machine [1] as an example case of a high-speed machine with a magnetically levitated rotor system. First, a system model with rigid dynamics is modeled, and then, a pole-placement-based state space control law is designed. After that, the parameter estimator properties as well as the adapting control functions are evaluated.

\subsection{Rigid system model with 4-DOF}

The AMB system with four degrees of freedom (4-DOF) as depicted in Fig. 4 is modeled in the bearing coordinates 
Table 1

Parameters of the Bearingless Machine

\begin{tabular}{lll}
\hline Symbol & Quantity & Value \\
\hline Rotor mass & $m$ & $11.65 \mathrm{~kg}$ \\
Rotor inertia & $I_{x}, I_{y}$ & $0.232 \mathrm{kgm}$ \\
Resistance, levitation winding & $R$ & $0.27 \Omega$ \\
Inductance, levitation winding & $L$ & $3.27 \mathrm{mH}$ \\
BM location & $a, b$ & $107.5 \mathrm{~mm}$ \\
Position sensor location & $c, d$ & $211 \mathrm{~mm}$ \\
Air gap length & $l_{\delta}$ & $0.6 \mathrm{~mm}$ \\
Rotor length & $l_{\mathrm{r}}$ & $480 \mathrm{~mm}$ \\
BM lamination stack length & $l_{\mathrm{rl}}$ & $61 \mathrm{~mm}$ \\
BM lamination diameter & $d_{\mathrm{rl}}$ & $68.8 \mathrm{~mm}$ \\
BM stator outer diameter & $d_{\mathrm{s}}$ & $150 \mathrm{~mm}$ \\
Rotor shaft diameter & $d_{\mathrm{rs}}$ & $33 \mathrm{~mm}$ \\
Current stiffness, measured & $K_{\mathrm{i}}$ & $29 \mathrm{~N} / \mathrm{A}$ \\
Position stiffness, measured & $K_{\mathrm{x}}$ & $672 \mathrm{~N} / \mathrm{mm}$ \\
\hline
\end{tabular}

using the position vector $q_{b}=\left[x_{A}, y_{A}, x_{B}, y_{B}\right]^{\mathrm{T}}$ with the input vector of currents $u=\left[i_{x A}, i_{y A}, i_{x B}, i_{y B}\right]^{\mathrm{T}}$. The dynamics of the system can be expressed in the following general form

$$
\mathbf{M} \ddot{\mathbf{q}}(t)+(\mathbf{D}+\Omega \mathbf{G}) \dot{\mathbf{q}}(t)+\mathbf{K q}(t)=\mathbf{F}(t),
$$

where $\mathbf{M}$ denotes the mass matrix with diagonal elements $\left[m, m, I_{x}, I_{y}\right], \mathbf{D}$ is the damping matrix, $\mathbf{G}$ is the gyroscopic matrix, $\mathbf{K}$ is the stiffness matrix, $\Omega$ is the rotational velocity, $\mathbf{F}$ denotes the forces applied to the rotor, and $\mathbf{q}$ is the displacement vector of the rotor. This model can be simplified to a rigid rotor model, which describes the rotor movement with respect to the center of the rotor mass. By simplifying the model and considering the bearing coordinates, the following model is obtained

$$
\mathbf{M}_{b} \ddot{\mathbf{q}}_{\mathbf{b}}+\Omega \mathbf{G}_{b} \dot{\mathbf{q}}_{\mathbf{b}}(t)=\mathbf{K}_{s} \mathbf{q}_{b}+\mathbf{K}_{i} \mathbf{u}
$$

where the position stiffness matrix is $\mathbf{K}_{s}=k_{s} \cdot \mathbf{I}$, and the current stiffness matrix $\mathbf{K}_{i}=k_{i} \cdot \mathbf{I}$ with $\mathbf{I}$ being a $4 \times 4$ identity matrix.

\subsection{Analysis of the approach}

The approach is studied by simulations using the values given in Table 1 and under the following conditions. The 11-cell PRBSs are injected to all the control inputs with an amplitude of $0.75 \mathrm{~A}$. The same amplitude has been used for SISO identification purposes in [1]. The poles of the position control are placed using a design function $z^{3}-2.8852 z^{2}+$ $2.7721 z-0.8869$, and the state observer is designed to be eight times as fast. The parameter estimation routine is initialized with the parameter matrix $\boldsymbol{\Theta}_{\text {init }}$ obtained from the values given in Table 1 . The covariance matrix is set to $F=10^{-6}$. I while $\lambda_{1}$ is set to 0.9997 . The controller is also designed based on the system values in Table 1, but the

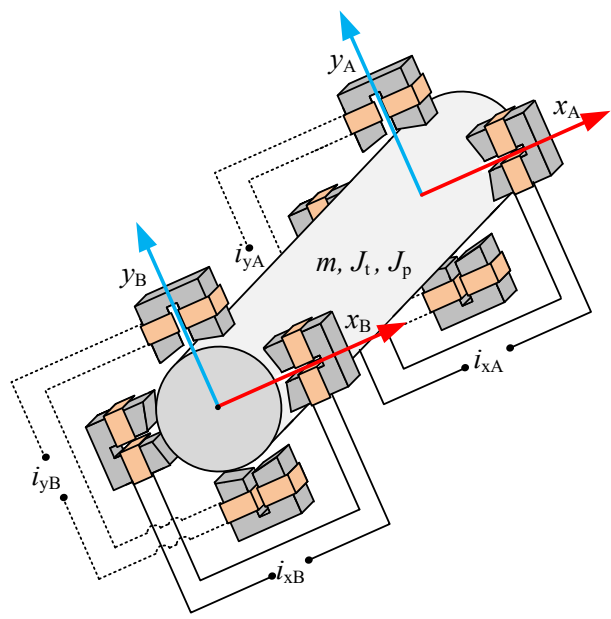

Figure 4: General principle of a rigid rotor suspended by two radial AMBs.

plant is modified for simulations to validate the self-tuning when the dynamics is not perfectly known.

First, the parameter estimation routine is validated in Fig. 5. Although the estimates are changing after the transient, as is expected in the case of recursion, the estimates are still stable after the routine is initiated at $0.1 \mathrm{~s}$. Owing to the symmetrical system dynamics, the estimates are close to each other. Next, the routine is connected with the control update routine, and the results are shown in Fig. 6. Based on the results, it can be observed that the transition phase from the fixed controller to an adaptive one is smooth, and more importantly, reasonable control parameters are estimated as the initial ones. Here, it is worth remarking that in the experimental section, an experimental 2-DOF AMB system is tested so that the initial model used for the control design deviates from the actual dynamics. Thus, for this special case, the proposed approach is capable of retuning the controller by adaptation.

\section{Experimental Results}

First, the approach is validated with an experimental 2DOF AMB system depicted in Fig. 7 a). The current sources for the bearing systems are standard ASCM1 industrial frequency converters manufactured by $\mathrm{ABB}$, and the adaptive control functions are implemented applying the Beckhoff TwinCAT environment with a Matlab/Simulink interface. The communication is obtained through an EtherCAT fieldbus, and the position is measured with 3300 XL NSV eddy current sensors by Bently Nevada.

\subsection{Open-loop self-tuning validation}

The controller update routine is first validated without closed-loop adaptation; to be more specific, the controller design functions are validated based on real-time estimated parameters. Moreover, the initial pole placement controller gains are designed in the initial mathematical model that is based on the physical assumption of the systems given in 

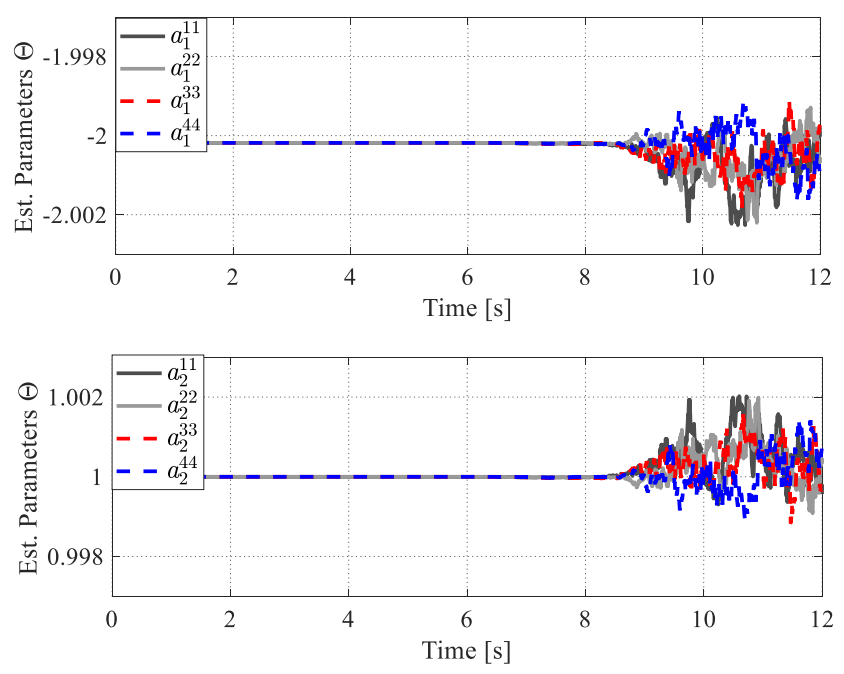

Figure 5: Parameter estimates during the open-loop identification corresponding with the subsystems on the diagonal of $\boldsymbol{\Phi}_{c}$.

Table 2. The parameter estimation routine is initialized with the same parameters $\boldsymbol{\Theta}_{\text {init }}$ and $F=10^{-6} \cdot \mathbf{I}_{12 \times 12}$ is selected to represent higher confidence for the unknown parameters. The controller is designed with (45) using $\omega_{n}=280, \zeta=0.6$ and $\tau=0.0015 \mathrm{~s}$. The amplitude of the PRBS is set to 0.2 A.

In Fig. 8, the results of the self-tuning of the state space controller gains $\mathbf{K}$ and $\mathbf{K}_{\mathrm{I}}$ are shown. The estimation is initiated at $1.3 \mathrm{~s}$, and the transition phase represents the parameter convergence transient when $\lambda_{1}$ is set to 0.9992 . The integral gain is validated by designing the controller using the whole identification experiment offline. The results show that the integral gains are updated to similar values as the offline-calculated ones, indicating that the online pole placement routine provides valid results. Furthermore, it can be noticed that the state feedback $\mathbf{K}$ is adapting and also showing cross-coupling terms (parameters in the middle). This result indicates that the self-tuning part can be straightforwardly adopted as part of the self-commissioning advancedmodel-based controller. To support this observation, in Fig. 9, the parameter estimates during the identification are shown. We can see that the transient is stable and the parameters converge to stable values.

Next, the open-loop self-tuning case is tested with the $10 \mathrm{~kW}$ bearingless prototype machine, shown in Fig. $7 \mathrm{~b}$ ), under full levitation. The same conditions for the parameter adaptation are considered as in the simulations above. The power electronics, sensors, and control software of the experimental system are similar to the 2-DOF system. The experimental results of controller adaptation are shown in Fig. 11. In this case, the initial conditions of the estimator correspond to the actual plant as in the simulations, and thus, the change in the controller gains is not significant. This result shows that online-identified model-based controller self-
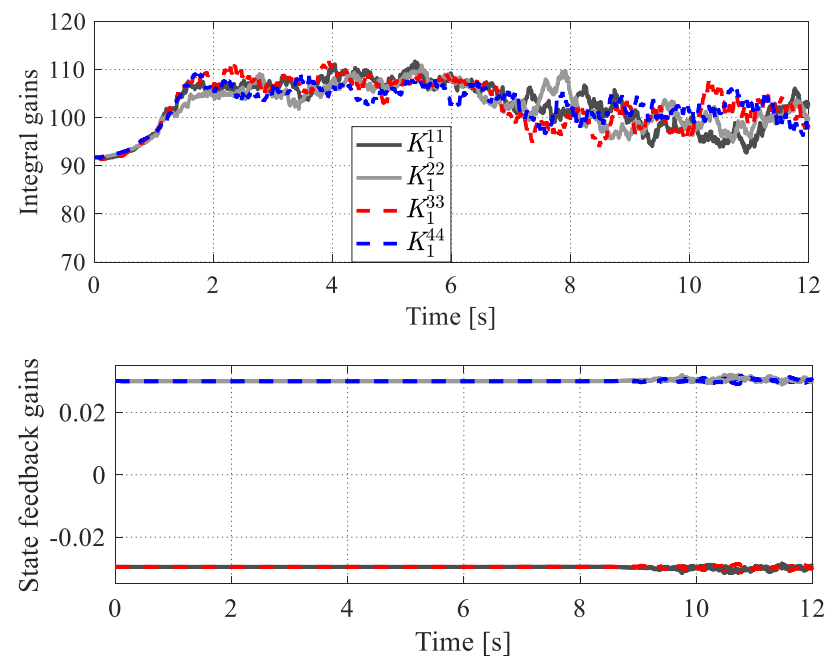

Figure 6: Adaptive control parameters; a) integral gains and b) state feedback gains.

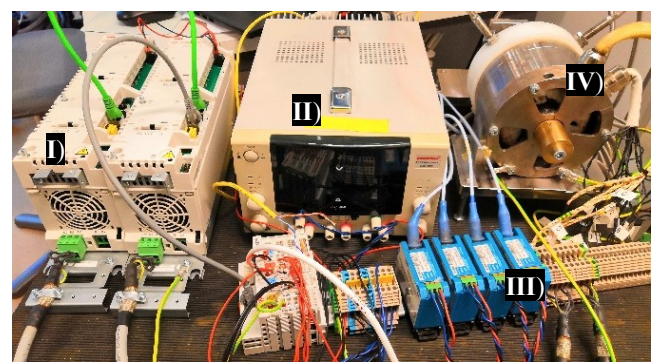

a)

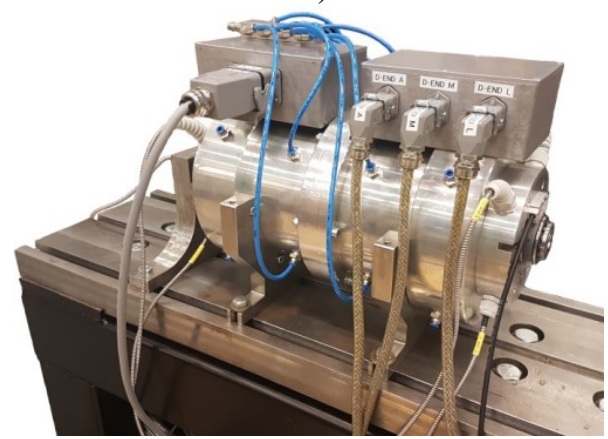

b)

Figure 7: a) Experimental 2-DOF AMB system; I) inverters, II) power supply, III) measurement amplifiers, and IV) AMB.

b) Bearingless $10 \mathrm{~kW}$ prototype machine.

commissioning or retuning is a reasonable option for a modern high-speed drive and the result corresponds well with the simulated one in Fig. 6.

\subsection{Closed-loop adaptation}

The adaptive state feedback pole placement law is validated by considering similar conditions and initialization as above in the case of the 2-DOF, but now the constant trace algorithm is applied and the excitation signal amplitude is 

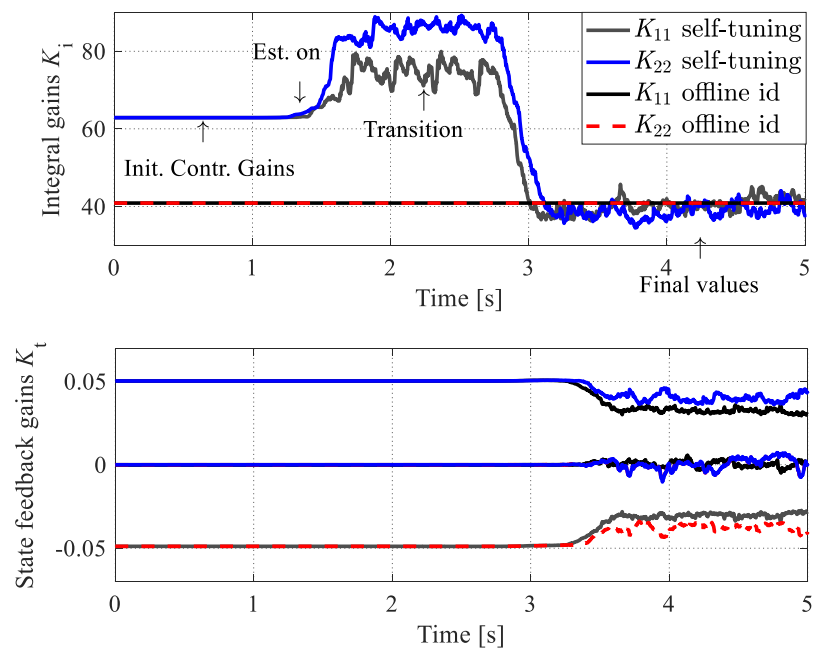

Figure 8: Experimental test of an open-loop self-tuning pole placement controller. The integral gains are shown in the upper figure and the state feedback gains in the lower figure.

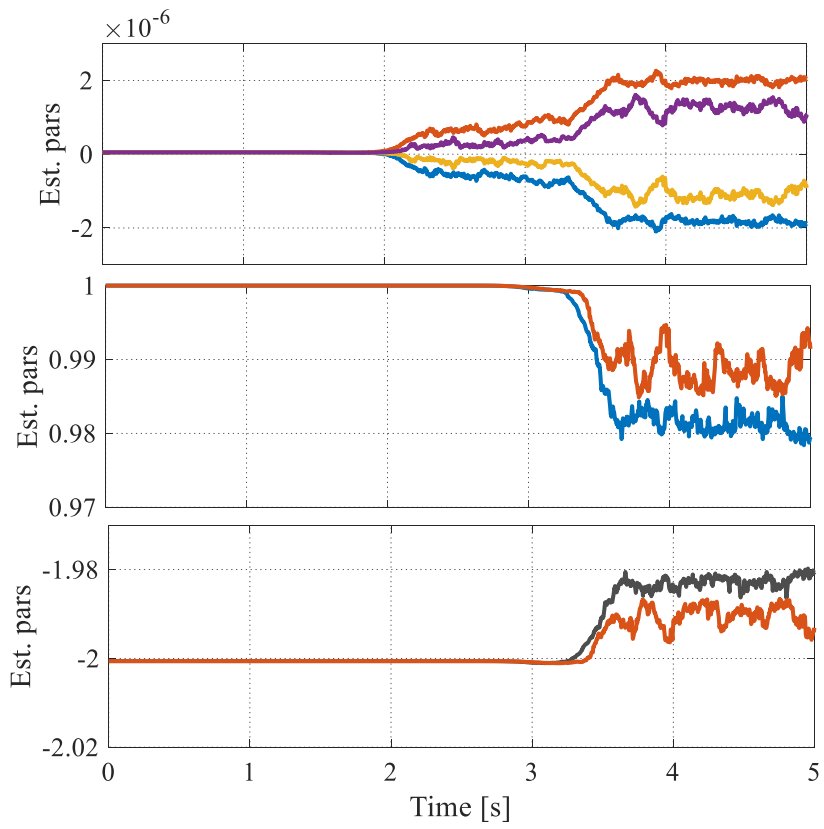

Figure 9: Parameter estimates during the open-loop selftuning test.

increased to $0.25 \mathrm{~A}$. The experimental results of the state space controller adaptation are shown in Fig. 10. It can be observed that after the adaptation is turned on, the transient is faster because of the tuning of the constant trace algorithm. More importantly, it can be seen that stable adapting controller parameters are obtained from the algorithm both for $\mathbf{K}$ and $\mathbf{K}_{\mathrm{I}}$.
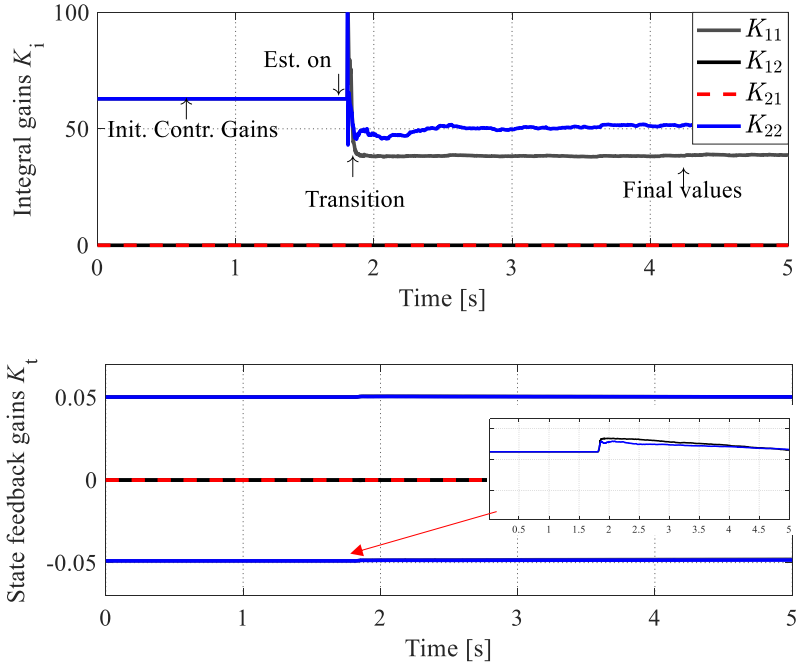

Figure 10: Experimental test of the closed-loop adaptive pole placement controller. The integral gains are shown in the upper figure and the state feedback gains in the lower figure.
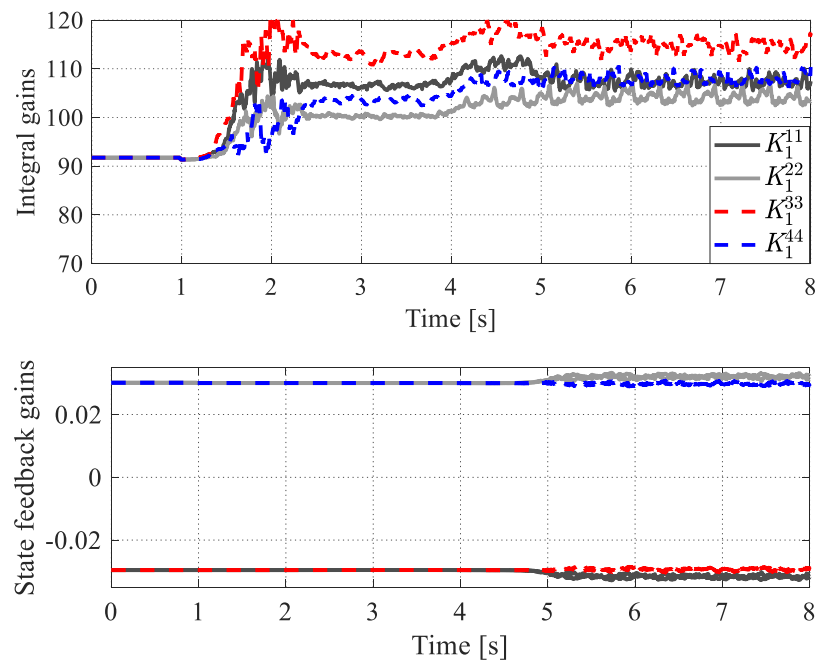

Figure 11: Experimental results of the open-loop self-tuning test in the 4-DOF case.

\section{Conclusions}

This paper studied the adaptive pole placement control of a high-speed machine with active magnetic bearings. An approach was proposed that is capable of adaptive self-commissioning based on a rigid model, thus giving more options for typically applied system identification offline commissioning steps. The identification approach was based on a recursive extended least squares estimator that was combined with the idea of using a canonical state space form for the controller and the state feedback gain update.

The experimental validation indicated that a complex adaptive MIMO control structure applied to AMB systems is ca- 
Table 2

2-DOF AMB System Parameters Based on Physical Assumptions

\begin{tabular}{lll}
\hline Symbol & Quantity & Value \\
\hline$k_{s}$ & Position stiffness & $3.7 \cdot 10^{5}[\mathrm{~N} / \mathrm{m}]$ \\
$k_{i}$ & Current stiffness & $61.4[\mathrm{~N} / \mathrm{A}]$ \\
$m$ & Mass & $1.52[\mathrm{~kg}]$ \\
$R$ & Coil resistance & $2.13 \Omega$ \\
$L$ & Coil inductance & $20[\mathrm{mH}]$ \\
\hline
\end{tabular}

pable of providing stable controller parameters under proper system identification conditions. It is noteworthy that the same approach can be modified so that the rigid controller part is a fixed baseline controller, and the adaptive part is considered for instance for flexible modes.

\section{References}

[1] P. Jaatinen, J. Vuojolainen, N. Nevaranta, R. Jastrzebski, and O. Pyrhönen. Control System Commissioning of Fully Levitated Bearingless Machine. Modeling, Identification and Control, 40(1):27-39, 2019.

[2] J. Shi, R. Zmood, and L. J. Qin. The direct method for adaptive feedforward vibration control of magnetic bearing systems. In 7th International Conference on Control, Automation, Robotics and Vision, 2002. ICARCV 2002., volume 2, pages 675-680 vol.2, Dec 2002.

[3] Min Xiang and Tong Wei. Autobalancing of high-speed rotors suspended by magnetic bearings using $1 \mathrm{~ms}$ adaptive feedforward compensation. Journal of Vibration and Control, 20(9):1428-1436, 2014.

[4] J. Kejian, Z. Changsheng, and C. Liangliang. Unbalance compensation by recursive seeking unbalance mass position in active magnetic bearing-rotor system. IEEE Transactions on Industrial Electronics, 62(9):5655-5664, Sep. 2015.

[5] Q. Chen, G. Liu, and B. Han. Suppression of imbalance vibration in amb-rotor systems using adaptive frequency estimator. IEEE Transactions on Industrial Electronics, 62(12):7696-7705, Dec 2015.

[6] M. L. Long, J. J. Carroll, and R. Mukundan. Adaptive control of active magnetic bearings under unknown static load change and unbalance. In Proceeding of the 1996 IEEE International Conference on Control Applications, pages 876-881, Sep. 1996.

[7] L. Dong and S. You. Adaptive control of an active magnetic bearing with external disturbance. ISA Transactions, 53(5):1410 - 1419, 2014.

[8] P. Wurmsdobler and H. P. Jörgl. State space adaptive control for a rigid rotor suspended in active magnetic bearings. In Proc. of the 5th Int. Symp. on Magnetic Bearings, pages 185-190, 1996.

[9] O. Lang, J. Wassermann, and H Springer. Adaptive vibration control of a rigid rotor supported by active magnetic bearings. ASME. J. Eng. Gas Turbines Power, 118(4):825-829, 1996.

[10] M. Hirschmanner and H. Springer. State space adaptive control for a rigid rotor suspended in active magnetic bearings. In Proc. of the 8th Int. Symp. on Magnetic Bearings, pages 483-488, 2002.

[11] D. Longand and L. Zongli. Control of a flexible rotor active magnetic bearing test rig: a characteristic model based all-coefficient adaptive control approach. Control Theory and Technology, 12(1):1-12, Feb 2014.

[12] F.K. Omani and N.K. Sinha. A modified state-space approach to multivariable self-tuning control with pole assignment. IEE Proceedings D, Control Theory and Applications, 134:31-37, Jan 1987.

[13] A. Unterholzner and $\mathrm{H}$. Wunsche. Adaptive state space control of a camera platform for an autonomous ground vehicle. In 2009 IEEE Intelligent Vehicles Symposium, pages 591-596, June 2009.

[14] Y. Wen-Shyong. An indirect adaptive pole-placement control for mimo discrete-time stochastic systems. International Journal of Adaptive Control and Signal Processing, 19(7):547-573, 2005.

[15] G. Messager and A. Binder. Observer-based pole placement control for a double conical high-speed bearingless permanent magnet synchronous motor. In 2016 18th European Conference on Power Electronics and Applications (EPE'16 ECCE Europe), pages 1-10, Sep. 2016.

[16] Yang Liu, Shuaishuai Ming, Siyao Zhao, Jiyuan Han, and Yaxin Ma. Research on Automatic Balance Control of Active Magnetic BearingRigid Rotor System. Shock and Vibration, 2019(1):1-13, 2019.

[17] C. Wagner, W. Tsunoda, T. Berninger, T. Thümmel, and D. Rixen. Estimation of rotordynamic seal coefficients using active magnetic bearing excitation and force measurement. In Proceedings of DINAME 2017, pages 3-15.

[18] A. Gambier and H. Unbehauen. Adaptive multivariable state-space receding horizon control of a turbo-generator pilot plant. In Proceedings of 35th IEEE Conference on Decision and Control, volume 4, pages 3688-3693 vol.4, Dec 1996.

[19] I.D. Landau, R. Lozano, M. M'Saad, and A. Karimi. Adaptive control: Algorithms, analysis and applications, chapter 3, pages 55-120. Springer, 2011.

[20] A. Gambler. Multivariable adaptive state-space control: A survey. In 2004 5th Asian Control Conference (IEEE Cat. No.04EX904), volume 1, pages 185-191 Vol.1, July 2004.

[21] L. Ljung and T. Söderström. Theory and practice of recursive identification, pages 125-128. MIT Press, London, 1987.

[22] J. Vuojolainen, N. Nevaranta, R. Jastrzebski, and O. Pyrhönen. Comparison of excitation signals in active magnetic bearing system identification. Modeling, Identification and Control, 38(3):123-133, 2017.

[23] Kurt E. Häggblom. Evaluation of experiment designs for mimo identification by cross-validation. IFAC-PapersOnLine, 49(7):308-313, 2016.

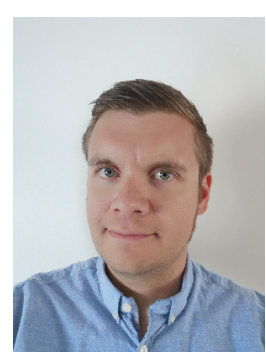

Niko Nevaranta received the B.Sc., M.Sc. and D.Sc degrees in electrical engineering from Lappeenranta University of Technology (LUT), Lappeenranta, Finland in 2010, 2011 and 2016, respectively, where he is currently working as a PostDoctoral Researcher. His research interest includes modeling and control of electrical drives, motion control, system identification, parameter estimation, system monitoring, and diagnostics. Currently he is also researching control approaches for active magnetic bearings and methods for rotor dynamics identification.

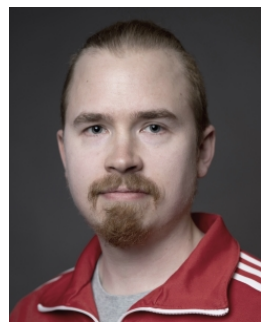

Pekko Jaatinen received the B.Sc. degree in electrical engineering from Saimaa University of Applied Sciences, Imatra, Finland, 2010, and the M.Sc. degree in electrical engineering from Lappeenranta University of Technology (LUT), Lappeenranta, Finland, 2013, where he is currently working toward the doctoral degree. His research interest include magnetic levitation, bearingless machines, and control systems.

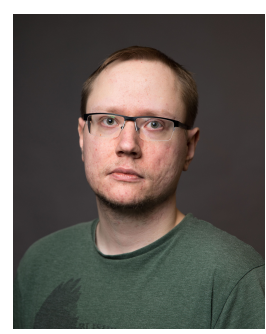

Jouni Vuojolainen received the B.Sc. and M.Sc. degrees in electrical engineering from Lappeenranta University of Technology (LUT), Lappeenranta Finland in 2014 and 2015, respectively, and is currently working toward his doctoral degree in electrical engineering. His current research interests include active magnetic bearing and rotor dynamics identification and application of identification methods to the diagnostics of rotating systems. 


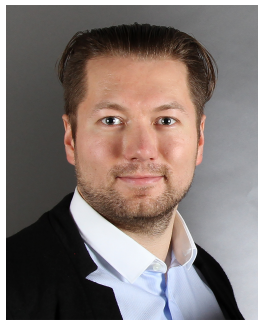

Teemu Sillanpää received the B.Sc. and M.Sc. degrees in electrical engineering in 2012 and 2013, respectively, from Lappeenranta University of Technology, Lappeenranta, Finland, where he is currently working toward the D.Sc. degree with the Control Engineering and Digital Systems Laboratory. His research interests include power electronic circuits, digital signal processing, and control systems related to active magnetic bearing suspended electrical machines.

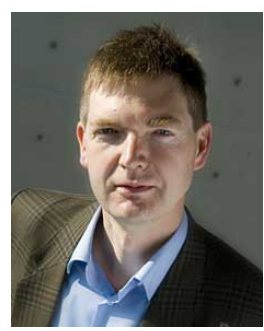

Olli Pyrhönen received the M.Sc. and D.Sc. degrees in electrical engineering from Lappeenranta University of Technology (LUT), Lappeenranta, Finland, in 1990 and 1998, respectively. Since 2000, he has been Professor in applied control engineering at LUT. In 2010, he received further teaching and research responsibility in the wind power technology at LUT. He has gained industrial experience as a RD Engineer with ABB Helsinki from 1990 to 1993 and as a CTO of The Switch from 2007 to 2010. His current research areas include modeling and control of active magnetic bearings, bearingless machines, renewable power electronics, and electrical drive systems. 